\title{
Educational Intervention for Effective Postoperative Pain Management in Low Resource Settings: Evidence from Ethiopia
}

\section{Million Tesfaye Eshete ( $\square$ mtesfaye1@gmail.com )}

Jimma University College of Public Health and Medical Sciences https://orcid.org/0000-0002-4994-354X

\section{Petra I. Baeumler}

Multidisciplinary Pain Center Department of Anesthesiology University Hospital Ludwig Maximilians

University (LMU)

\section{Markos Tesfaye}

St. Paul's Hospital Millennium Medical College

\section{Yemane Ayele}

Department of Anesthesiology Institute of Health Faculty of Medicine Jimma University

\section{Abraham Haileamlak}

Department of Pediatrics and Child Health Institute of Health Faculty of Medicine Jimma University

\section{Girma G Michael}

Department of Anesthesiology Institute of Health Faculty of Medicine Jimma University

\section{Dominik Irnich}

Multidisciplinary Pain Center Department of Anesthesiology University Hospital Ludwig Maximilians University (LMU)

\section{Matthias Siebeck}

Center for International Health Medical Center of the University of Munich (LMU) Germany and Department of General Visceral and Transplantation Surgery Medical Center of the University of Munich (LMU)

\section{Research Article}

Keywords: Postoperative pain, Education, Ethiopia, Quasi-Experiment

Posted Date: January 3rd, 2019

DOI: https://doi.org/10.21203/rs.2.171/v1

License: (9) This work is licensed under a Creative Commons Attribution 4.0 International License. Read Full License 


\section{Abstract}

\section{Background}

The annual number of surgical operations performed is increasing throughout the world. With this rise in the number of surgeries performed, so too, the challenge of effectively managing postoperative pain. Healthcare professionals and patients in education might help in controlling postoperative pain effectively. However, data from low-income countries investigating the impact of educational intervention on postoperative pain are very scanty, and reports from the developed settings are inconclusive. The study has investigated the impact of preoperative patient education and health care professionals education on improving the quality of postoperative pain management; in patients scheduled for major elective orthopedic, gynecologic and general surgery; as measured by patient-reported outcomes.

\section{Methods}

This was a quasi-experimental, non-equivalent control group design with before and after measures. We have recruited 700 consecutive patients; who are eighteen years or more, scheduled for general, orthopedic and gynecologic surgery. Outcome measures were patient-reported outcomes (postoperative pain intensity, pain interference, and perception of care) and adequacy of pain management used.

Results

Generally, no significant difference was observed in most outcome measures except for the worst level of pain, least level of pain, patient participation in decision making and feeling of drowsiness between the treatment and control group.

\section{Conclusion}

Results of this study contained very important information in understanding the effectiveness of educational interventions in the postoperative setting. The treatment was successful in increasing patients participation in decision making, as anticipated. However, its impact on decreasing pain intensity was only noted at the last measurement point after surgery.

\section{Background}

The annual number of surgical operations performed is increasing throughout the world. It is estimated that in the year 2012 alone, 266.2 to 359.5 million operations were performed. This figure has raised by $38 \%$ compared to the previous eight years and low-income countries are contributing to the greater share [1]. Also, the number of deaths as a result of surgery is twice the number of maternal deaths per year [2]. It has been estimated that about $22 \%$ of chronic pain is caused by surgery [3]. Yet chronic pain exceeds the economic burden of cardiovascular diseases and cancers combined [4]. Acute pain after surgery also remains to be a challenge in the globe [5] and up to $40 \%$ of patients suffer from severe pain intensity [6]. Untreated or undertreated postsurgical pain can lead to prolonged hospital stay, persistent postoperative pain, respiratory and cardiovascular 
complications, anxiety, depression and even premature death [7, 8]. Developing countries while fighting to eradicate poverty, hunger, reduce maternal and child mortality; pain management is abandoned and the global health policy is turning a blind eye to the aftermath of uncontrolled pain and its global burden [9]. Though remains to be a problem for both developed [6] and developing countries [10]; sadly the suffering from untreated pain is larger and troublesome amidst the poor [9]. Hence, for countries like Ethiopia, a cost-effective effective intervention is mandatory.

Various strategies have been attempted to rescue the patient from suffering undertreated and untreated postsurgical pain until now [11]. As early as 1958, Fink et al. have reported the advantage of preoperative educational intervention as one of the strategies to reduce postoperative pain [11]. Following this many more studies in the field tried to replicate the findings. However, some authors reported no effect of preoperative patient education after conducting a well-randomized controlled trial [12]; while others claim a positive effect on postoperative pain. Lately, however, the argument whether preoperative patient education is effective or not is starting to materialize in literature[13]. A systematic review and meta-analysis of RCT on the topic also failed to bring consistent results; while some support [14] and others not [15]. In some studies, patients received various forms of other therapeutic interventions along with the preoperative education; authors call this adjunct treatment [16]. Popular adjunct treatments that accompanied preoperative education included either exercise or relaxation [16]. As distinguished from studies performed until now, we have accompanied the preoperative patient education by Healthcare Providers (HCPs') education; by hypothesizing a well educated patient and $\mathrm{HCP}$ can perform better synergistically. A review of literature recommends educational programs to include interdisciplinary professionals and policymakers in addition to patients [17].

The aim of this study was to investigate the impact of preoperative patient education and HCPs education on postoperative pain outcome in patients scheduled for major elective orthopedic, gynecologic and general surgery. In addition to testing the impact of the intervention on the outcome; unlike to the previous studies, we have attempted to explain the underlying mechanisms of the preoperative education. Hitherto, it is believed that providing patients with education is to address preoperative level of anxiety in the preoperative period and then subsequently to decrease pain intensity [16]. All experimental studies so far tested whether the education is effective or not in decreasing patient pain intensity and no study attempted to verify if, in fact, preoperative education affects patient postoperative pain through anxiety or any other pathway. One of the emerging statistical tools to understand the causal mechanism behind an intervention is causal mediation analysis [18]. With the help of this method, we have attempted to answer whether the designed intervention was effective and also explain the underlying mechanism. So, this study provides information regarding the effectiveness of preoperative patient education on postoperative pain and also the causal mechanism.

\section{Materials And Methods}

This was a quasi-experimental, non-equivalent control group design to assess the effectiveness of educational intervention given to patients and HCPs. The study was conducted in three large teaching hospitals located, in two regions of Ethiopia. The two hospitals (Zewditu Memorial Hospital Medical college and Yekatit 12 Hospital) were assigned to the control group and one (Jimma University Teaching Hospital) to the intervention. The control hospitals are located in the capital city Addis Ababa and the intervention hospital is $350 \mathrm{~km}$ away from the capital in south-west Ethiopia, Oromia region. The experimental hospital (group) and the control 
hospitals (group) were determined by geography. Both groups were pretested simultaneously, before the administering of the treatment. At the end of the treatment, a posttest was administered simultaneously to both groups. The study was performed in accordance with ethical standards established in the 1964 Declaration of Helsinki, and the protocol was approved by the ethics committee at the Jimma University (Ref.No RPGC/06/2016; Jimma, Ethiopia) and the Ludwig Maximillian University of Munich Medical Ethics Committee (Ref. No 17-224, Munich, Germany). All participating hospitals also granted permission for the study in

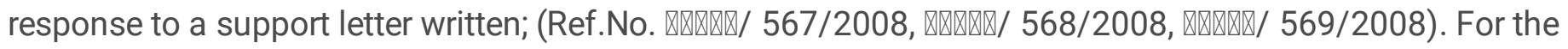
illiterate participants (those who could not read and write) their fingerprints were obtained as an indication of their consent after the information sheets and consent forms were read aloud by the data collectors. Patients who provided written consent to participate completed an interviewer-administered baseline questionnaire, prior to the intervention (September to December 2016) and after the intervention (May to August 2017). Patientreported outcomes were measured at four-time points postoperatively at 6, 12, 24 and 48 hours). After the baseline assessment HCPs and hospital officials of the experimental group were invited to participate in an educational intervention especially designed for the group. Thirteen participants from nursing, physiotherapy, surgery, anesthesiology, gynecology schools including those in the administrative position participated following the invitation. Post-treatment assessment was performed 19-20 weeks after baseline assessment (May-August 2017).

\section{Participants}

The night before the planned operation we have identified eligible patients from the surgical waiting list. Next, we have approached them to explain the study objectives and expected the role of participation. Follow-up was initiated after patients approved participation by signing the informed consent. We have analyzed 700 consecutive patients; who are eighteen years or more, scheduled for general, orthopedic and gynecologic surgery. Those having cognitive and mental disabilities (identified from their clinical record files), patients transferred directly to an intensive care unit, those who had emergency surgery including cesarean section were excluded. Details are provided in Figure 1 with participants flowchart.

\section{Intervention}

The contents of all training materials were based on literature review $[13,16,17,19]$ IASP recommendations [20], international recommendations for low resource settings[21, 22] national guidelines [23] and considering the principles of learning sciences. The latter takes into account the conditions, processes, and outcomes of learning [24]. Before the main preoperative patient education, staff members of the intervention hospitals were trained on the effective management of post-surgical pain. Health care providers including those who assume a leading or managerial position, surgeons, gynecologists, nurses, and physiotherapists were invited to participate. A total of 13 participants (3 anesthetists, 3 surgeons, 2 gynecologists, 1 physiotherapist and 4 nurses) attended the workshop. For about 13 hours over 3 days, they were trained on topics related to the obstacles to pain management in low-resource settings, the importance of pain assessment, measurement, and tools, use and application of non-pharmacological methods of pain management. In addition to the theoretical lectures, participants were exposed to the practice sessions. The hands-on sessions focused on the use of nonpharmacological methods of pain management with emphasis on acupuncture. 
After the HCPs education was completed, the night before the surgery, a project team member an anesthetist conducted a one-on-one education verbally. Each session lasted for 15 minutes. Voluntary relatives also attended. In brief, each educational session consists of information regarding why managing postoperative pain is important, non-pharmacological options of pain management, how to take pain medication as directed, report side effects early, participate in the choice of the management of pain with HCPs. Patients were informed that they should not be shy and always be active in the management of their pain. They were also told how to describe their pain using the pain intensity scales. During the educational session, patients were encouraged to ask questions. Once the question and answer session was completed, each patient was asked to repeat what they have learned. Finally, five questions were asked of all patients and if a patient misses one question education was repeated again. Patients in the control hospitals received care as per usual with no preoperative education. Preoperative education or information for postoperative pain is not part of the care in the setting yet.

\section{Table 1. Components of the patient educational intervention}

\section{Topic Contents and Evidence \\ Covered}

Introductions

Patient and his/her families greeted warmly

What is about to be thought introduced and

All are asked to sit comfortably.

Overview of Definition of post-surgical pain, what causes it and how can it be managed post-surgical

pain

Goals of Why managing post-surgical pain is important and Highlighting the management consequences of unmanaged postoperative pain [19]

\section{Patient role The patient should ask for analgesics and insist if the health care provider is in the not responding, Patients should not be passive but actively participate in management decision [8, 25, 26,27$]$. How to take medications as directed, manage side- effect early, avoid misconception [28] report side-effect early [29] communicate your pain using instruments [30] \\ How to be relaxed and avoid fear prior to surgery \\ Should believe that unmanaged pain is very harmful [19] \\ Available Both pharmacological and non-pharmacological methods [19] \\ options for How to reduce anxiety using various alternatives [31] \\ treatment}

The night before the operation, the trained data collectors from all sites approached potentially eligible patients. Next, patients were provided with a detailed information sheet describing the study and their potential 
involvement. For the majority of the patients (who were illiterate), the written information sheet was read to them aloud. But, in any case, a witness's signature was obtained. Patients who provided written (fingerprint in case of the illiterate) and verbal consent to participate completed an interviewer-administered baseline questionnaire, prior to the intervention

Patients were aware of their participation in a study but were blind to which condition the hospital was allocated. However, those who administer the interventions and those assessing outcomes were not blind to study allocation. All evaluations were conducted in the participants' surgical wards using a paper and interviewer-administered questionnaire. Data collectors were different from those who administered the intervention. During the intervention period, however, in addition to the offer to participate in the intervieweradministered self-reported measures, patients in the treatment group were also given additional information about the planned preoperative education. When the patient agreed to participate, a consent was obtained the same way as described above and the preoperative individualized patient education was conducted. Consequently, using the interviewer-administered questionnaire, patient-reported outcomes were collected after the operation at the four-time points explained above.

\section{Outcome measures}

The main outcome measure was measured using the Numeric Rating Scale (NRS 0-10; $0=$ No pain $-10="$ Worst pain"). All secondary outcome measures were measured using the IPOQ (International Pain Outcome Questionnaire)-originally developed from the American Pain Society Patient Outcome Questionnaire (APSPOQ) [32]. It has been translated into 15 different languages and validated in 8 European countries and Israel. It includes questions on pain severity, pain interference with physical function and emotions, side effects of pain treatment, and perception of care. Also, it permits to grasp information about the use of nonpharmacological methods for pain relief and the presence of preoperative chronic pain. IPOQ items mostly use 11-point (NRS 0-10) numeric rating scale, but also binary items are included. Patient worst, least and current pain intensity was measured as (NRS $0=$ "no pain" $-10=$ "worst pain possible." The percentage of time the patient spent in severe pain since surgery was measured as $0 \%=$ "never in severe pain" $-100 \%=$ "always in severe pain." Pain interference was measured as functional disability due to pain (NRS $0=$ "did not interfere"$10=$ "completely interfered"), anxiety and helplessness caused by pain (NRS $0=$ "not at all" $-10=$ "extremely"). Patient perception of care was measured as the degree of pain relief through pain treatment (NRS: $0 \%=$ "no relief"- $100 \%$ = "complete relief"). Patients wish for more analgesics were recorded as binary ("yes or no") answers. Satisfaction with the results of pain treatment was measured with NRS $0=$ "extremely dissatisfied"$10=$ "extremely satisfied." The original English version has been translated (forward and backward) into two local languages and pilot tested in five steps as per international guidelines [33]. The final version was approved by expert panel to make sure content and face validity. In addition, we have measured the adequacy of pain management using the Pain Management Index (PMI). The index is calculated by first categorizing patients worst pain intensity into 0 (no pain), 1 (1-3: mild pain), 2 (4-6: moderate pain), and 3 (7-10: severe pain). The final score is then subtracted from the strength of analgesic prescribed: which is 0 (no analgesic drug), 1 (non-opioids), 2 (weak opioids), and 3 (strong opioids). The final score is between -3 to +3 , and negative scores inform inadequate treatment. Originally this was designed to assess the adequacy of cancer pain management; however, its application in surgical patients have been reported [34]. 


\section{Covariates}

The covariates considered in this study were the following: time (since surgery), patient's age and sex, preexisting chronic pain, patient's physical condition and operating time. We have also retrieved demographics, medical history information, type of surgery, duration of surgery, type of anesthesia and pain treatment from the medical records.

\section{Statistical analysis}

Before testing the effectiveness of the intervention, we calculated mean and SD for normally distributed continuous variables and medians ( $\mathrm{min}, \mathrm{max}$ ) in case of skewed distributions. Categorical variables were summarized as numbers (percent). In order to assess the influence of selection bias, differences in baseline clinical and demographic variables at the baseline were evaluated using univariate generalized linear models and using Chi-square test. Comparison of changes in the outcomes of interest over time between the control and treatment group were analyzed using linear mixed effect model. Covariates in the final model were selected using backward elimination, which begins with the maximum full model and then deleting variables of no value. However, age, sex, type of surgery, and chronic pain severity were left in the model despite statistical insignificance, to avoid omitting a significant variable (avoid any Type II errors) and therefore maximize validity and predictive power, which is a good practice [35]. To adjust for baseline differences between the treatment and control groups, in addition to the baseline outcome value, we have also added additional fixed terms to the LME model. These include age, sex, type and duration of surgery, type of anesthesia, ASA-classification and preoperative chronic pain severity. In the final model, two random effects were included: the random intercept and the random slope of time (that is, number of hours after the operation) for patients.

\section{Sensitivity analysis.}

To test how robust our findings are we conducted a sensitivity analyses using propensity score analysis[36, 37]. Robins et al., in 1994, proposed the doubly robust (DR) estimator, which is an amendment of the IPW methods [38]. This method brings together both the outcome regression model and the propensity score model. For this reason, the investigator has two opportunities (chances) of specifying the model correctly. Even if either the propensity score model of the outcome regression model misspecified, the DR remains consistent [38, 39]. A proper weighting and correct propensity score model removes confounding with respect to the measured baseline covariates, and thus, the average treatment effects obtained reflect the true population average [40]. Using a DR approach can compensate for a lack of covariate balance, unlike to other matching techniques of the propensity score. Moreover, with other previously mentioned matching techniques, the dataset can be preprocessed by "trimming" away (removing) individuals with extreme PS, while attempting balance [41]. Therefore, this method of estimation was used in this particular study, to calculate the average treatment effect.

\section{Causal Mediation analysis}

In the present study we have implemented the within-subject 1-1-1 multilevel mediation, also known as lower level mediation [42], page 179]. In longitudinal, within-subject mediation, $X, M$, and $Y$ can vary either withinsubjects (level-1), between-subjects (level-2), or both [42]. Krull and MacKinnon outlined three specific multilevel 
mediation scenarios: $2 \rightarrow 2 \rightarrow 1,2 \rightarrow 1 \rightarrow 1$, and $1 \rightarrow 1 \rightarrow 1$ [43]. Since the mediator (patient participation in decision making) is a level-1 variable and the treatment exposure was also individualized patient education, which is also a level-1 exposure and the outcome variable is also measured at level 1 (patients' worst pain intensity), we have conducted a $1 \rightarrow 1 \rightarrow 1$, within-subject mediation. We have followed the procedure described by Bolger and Laurenceau [42]. We have performed 1000 sample bootstrap procedure to estimate $95 \%$ confidence intervals (Cls) to test the significance of indirect links and Cls are expected not to contain 0 and only then the indirect links are considered to be significant [44]. The mediation analysis was also adjusted for all measured baseline confounders.

The treatment condition (treated vs control) is represented by $X$, patients participation in decision making is labeled $M$, and patients' rating of worst pain intensity (patients' satisfaction for the second mediation model) is labeled $Y$. The total effect was calculated using the formula from Kenny, Korchmaros, and Bolger[45] which is given by:

Here we see that $c$, the relationship between $X$ and $Y$ for the typical patient, is equal to the sum of (1) $a b$, the product of the $X$-to- $M$ and the $M$-to- $Y$ coefficients for the typical subject; (2) $c^{\prime}$, the coefficient representing the unmediated portion of the $X$-to- $Y$ relationship for the typical subject; and (3) sajbj, the covariance of betweensubjects differences in the $X$-to- $M$ and $M$-to- $Y$ relationships. Including the final covariance term 0 is very important in multilevel mediation and it has an important implications for estimates of mediated effects. It represents that the extent that those patients whose participation in decision making score is most affected by the treatment are the same patients whose pain intensity (patients' satisfaction for model 2) is most affected by their participation in decision making, then the overall mediated effect will be greater than one would expect from the $a b$ product alone [42]. All data management, linear mixed model building, and propensity score weighting was done using STATA version 13.0 (StataCorp., Texas, USA). For the multilevel causal mediation analysis, we have used the $\mathrm{R}$ function indirectMLM.R, written by Elizabeth Page-Gould [46]in R package version 1.3.4 in R Statistical Software (version 3.4.3; R Foundation for Statistical Computing, Vienna, Austria). Withinsubject mediation, by allowing between-subjects heterogeneity in mediated paths, affords a realistic conceptualization of psychological and interpersonal processes.

\section{Results}

First, we have assessed the psychometric properties of the IPOQ in terms of construct validity, internal consistency, and factor structure. Before exploratory factor analysis, the Kaiser-Meyer-Olkin (KMO) test and Bartlett's test of sphericity were conducted to evaluate the factorability. The KMO measure of sampling adequacy was 0.8587 and the significance of Bartlett's test of sphericity was less than 0.000 , meaning that EFA can be applied to the obtained dataset [ref]. Principal component analysis with varimax rotation was used. The factor analysis generated a four-factor solution (Eigenvalue $>1$ ), explaining a total variance of $64.8 \%$. The overall internal consistency of the IPOQ in our sample, based on Cronbach's alpha among all items, was 0.86 . Regarding IPOQ sub-scales, all four present acceptable values. The pain intensity and physical interference

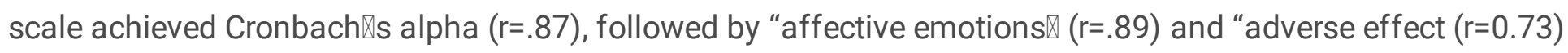
"perceptions of care $\mathbb{Z}(r=.62)$. All the above parameters were consistent and very much comparable with the reports of the original authors [37], except for the four-factor solution where the original authors reported 3factor structure. However, the phase-one data of the original authors reported four-factor solution with a total explained variance of $60.78 \%$ [37, page $=1368]$, which is consistent with our findings. As it is a common practice 
in the field to do so [37], discriminant validity was assessed by comparing surgical category of patients. We used Mann-Whitney $U$ tests and chi-square tests to contrast groups. Because of a small proportion of orthopedic and gynecologic patients, the two were combined together and contrasted with the general surgical patients. Except for least pain intensity, pain interference with sleeping, pain interfering with activities out of bed, patient perceived pain relief, and patient satisfaction, for all 12 NRS items a significant difference between the general surgery and comparative (orthopedic and gynecologic patients combined) groups was observed. Almost all (except the percentage of time patient spent in severe pain) pain intensity items, both items on affective impairment and 2 interference items, were significantly increased in the group where orthopedic and gynecologic patients were combined. All 4 adverse effects measures were also increased in the same group of patients.

\section{Participants' Characteristics}

Figure 1 shows the study flowchart. No baseline measures were balanced across the treatment and control groups. Rather, patients were significantly more likely to have been assigned to the intervention group if they are older, were more illiterate, Muslim, are married, are Oromo by ethnicity, had an orthopedic and gynecologic surgery, had a less duration of surgery, and lower chronic pain intensity (Table 1).

Table 2: Baseline characteristics of the sample by condition (Intervention and Control Group).

\begin{tabular}{|c|c|c|c|}
\hline & Control & Treated & p-alue \\
\hline Age, mean (SD) & $40.52(15.9)$ & $37.69(17.2)$ & $<0.001$ \\
\hline \multicolumn{4}{|l|}{ Sex } \\
\hline Male, n (\%) & $241(58.3)$ & $123(43)$ & $<0.001$ \\
\hline \multicolumn{4}{|l|}{ Educational Status } \\
\hline Literate, n (\%) & 324 (69.7) & $125(53.2)$ & $<0.001$ \\
\hline \multicolumn{4}{|l|}{ Religion } \\
\hline Orthodox, n (\%) & $349(75.1)$ & $77(32.8)$ & $<0.001$ \\
\hline \multicolumn{4}{|l|}{ Marital status } \\
\hline Married, n (\%) & $331(71)$ & $177(75.3)$ & 0.015 \\
\hline \multicolumn{4}{|l|}{ Ethnicity } \\
\hline Oromo, n (\%) & $112(24.1)$ & $156(66)$ & $<0.001$ \\
\hline \multicolumn{4}{|l|}{ Types of Surgery } \\
\hline General, n (\%) & $378(81.3)$ & $113(48.1)$ & $<0.001$ \\
\hline \multicolumn{4}{|l|}{ Types of Anesthesia } \\
\hline General, n (\%) & $350(75.7)$ & 158(67.5) & $<0.001$ \\
\hline \multicolumn{4}{|l|}{ ASA-Physical Status } \\
\hline $\mathrm{I}, \mathrm{n}(\%)$ & 459 (98.7) & $212(90.2)$ & $<0.001$ \\
\hline Duration of surgery in hours, mean (SD) & $1.85(0.9)$ & $1.47(0.9)$ & $<0.001$ \\
\hline Chronic pain severity, mean (SD) & $4.9(2.7)$ & $2.5(2.4)$ & $<0.001$ \\
\hline
\end{tabular}

Fig 1. Participant's flow chart. Phase1 is the time before the intervention (Bassline), and phase 2 is the time after the baseline. The gap between phase 1 and phase 2 here is about 20 weeks.

\section{The effectiveness of the intervention}


Generally, both the weighted and unweighted models gave consistent results for all pain intensity measures except for patients' worst pain intensity and for all the pain interference measures except for pain interference with sleeping and pain causing the feeling of anxiousness [see Additional file 1]. The interaction Group (treatment vs. Control) $x$ Time (6, 12, 24 and 48 hours) was significant for most outcome measures, implying the groups differed in rate and manner of change over the course of the study. Patients in the treatment group had scored lower worst pain intensity score at the second $(\beta=-1,95 \% \mathrm{Cl}(-1.649$, $-0.359)$ ), third ( $\beta=-1.553,95 \% \mathrm{Cl}:(-2.23,-0.875)$ ) and fourth $(\beta=-2.000,95 \%$ $\mathrm{Cl}:(-2.822,-1.178))$ measurement points respectively. However, in the weighted model, significant changes were observed at the third and fourth measurement points. Both weighted and unweighted model revealed that patients in the treatment hospital had a lower score of the percentage of time patient spent in severe pain at the last measurement point $(\beta=-0.80,95 \% \mathrm{Cl}:(-1.25,-0.35))$. The same consistent results were obtained between the weighted and unweighted models for both least and current level of pain at the fourth measurement points $(\beta=-0.73,95 \%$ $\mathrm{Cl}=(-1.21,-0.24)$ and $(\beta=-1.34,95 \% \mathrm{Cl}:(-2.38,-0.31))$ respectively. The treatment group had lower pain interference with activities in bed score at the second $(\beta=-0.9095 \% \mathrm{C} . \mathrm{I}$ : $(-1.46,-0.34))$, third $(\beta=-1.00,95 \% \mathrm{C}$. I : $(-1.75,-0.25))$ and fourth $(\beta=-1.89,95 \%$ C.I $(-2.78,-1.01))$ time points. Pain interfere with movement was improved in the treatment compared to the control $(\beta=3.13,95 \% \mathrm{Cl}:(-4.63,-1.63)),(\beta=-3.14,95 \% \mathrm{Cl}:(-3.94,-2.35))$, $(\beta=4.19,95 \% \mathrm{Cl}:(-5.22,-3.17))$ at the second, third, and fourth measurement points respectively. Pain interference with breathing and coughing was also significantly lower in the treatment group at the third and fourth measurement points $(\beta=-0.73,95 \% \mathrm{Cl}:(-1.30,-0.15)),(\beta=-1.26$, $95 \% \mathrm{Cl}:(-1.87,-0.64)$ respectively. However pain interference with sleeping was not significantly different between the two groups in the weighted model, and only at the last measurement point in the unweighted model. The treatment also lowered pain causing the feeling of anxiousness at the last measurement point $(\beta=-0.94,95 \% \mathrm{Cl}:(-1.59$, $-0.28)$ ) in the weighted model and in the second and last measurement point in the unweighted model. Consistent results were observed for the score patients' feeling of helplessness, where the treatment group has lower score at the last measurement points $(\beta=-0.84,95 \%$ C.I : $(-1.43$, 
-0.25)). Patient participation in decision-making was significantly higher in the treatment group at the second measurement points only $(\beta=3.81,95 \%$ C.I : $(2.69,4.93))$. Patients' satisfaction with the treatment remained unaffected by the treatment. The proportion of patients in the intervention group who were inadequately treated declined over time except at $\mathbf{4 8}$ hours before the intervention. Before the intervention, about $87 \%$ of patients were inadequately treated, however, after the intervention $55 \%$ of patients were inadequately treated at 6 hours after the surgery in the treatment group. The same way before the intervention about $72 \%$ of patients were inadequately treated in the treatment group and it dropped to $46 \%$ after the intervention. However, the proportion of patients inadequately treated increased from $30 \%$ to $41 \%$ and from $1 \%$ to $23 \%$ at the 24 and 48 hours after the surgery respectively. The same trend was observed in the control group that patients inadequately treated increased at the $\mathbf{2 4}$ hours and $\mathbf{4 8}$ hours. Both before and after the treatment patients in the treatment group were inadequately treated. After the treatment, about $70 \%$ of patients also received acupuncture treatment for postoperative pain in the intervention group.

\section{Mechanism of the intervention}

\section{Path a, of both figure 2 and 3}

The purpose of the first mediation analysis was, to examine the role of the educational intervention $(X)$ on postoperative pain intensity $(Y)$ through the mediating pathway of patient participation in decisionmaking $(M)$. The indirect, direct and total effects of each of the model are given in Figure 2. For the typical patient in the treatment group, there is clear evidence that the treatment $(X)$ predict greater participation in decision-making (M). Compared to the control group patients in the intervention group had a predicted 3.07 unit higher participation in decision making, 95\% Cl:(2.69, 3.46). Even after adjusting for measured covariates including age, sex, type of surgery, type of anesthesia, baseline worst pain intensity and duration of surgery, path a, remained significant and treatment predicted 2.4 units higher participation in decision making $95 \% \mathrm{Cl}:(1.972,2.707)$. 
Fig 2: Within-subject mediation (see Bolger and Laurenceau, 2013):

Diagram and structural equations. To reduce confusion, we have omitted time as a predictor and we treat $\mathrm{X}, \mathrm{M}$, and $\mathrm{Y}$ as varying within-subjects only. *Adjusted for age, sex, preoperative pain, type of surgery, type of anesthesia, baseline worst pain intensity and duration of surgery.

\section{Patients' participation in decision making on pain intensity: Path $b$, of figure 2}

The patient participation $(M)$ to postoperative pain intensity $(Y)$ slope for the average patient is $-0.0695 \% \mathrm{Cl}:(-0.19,0.08)$, indicating that, for patients in the treated group for each additional unit increase in decision making, it did not predict reduced postoperative pain intensity.

\section{Patients' participation in decision making on satisfaction: Path $b$, of figure 3}

The unadjusted patient participation (M) to patient satisfaction (Y) slope for the average patient is $0.227,95 \% \mathrm{Cl}:(0.125,0.369)$, indicating that, for patients in the treatment group with each additional unit of patient participation in decision making, it predicted a higher satisfaction. However, when adjusted for baseline confounders the result is insignificant $0.01895 \%$ C.I $(-0.293,0.267)$.

Figure 3: Within-subjects mediation for satisfaction (see Bolger and Laurenceau, 2013): To reduce confusion, we have omitted time as a predictor and we treat $X, M$, and $Y$ as varying within-subjects only. *Adjusted for age, sex, preoperative pain, type of surgery, type of anesthesia, baseline worst pain intensity and duration of surgery.

The indirect effect Path $a * b$, for Figure 2

The indirect effect (Path $a * b)$ of treatment on postoperative pain intensity, with patient participation in decision making as the potential mediator, was not statistically significant for both, the unadjusted $a b=-0.106,(95 \% \mathrm{Cl}:(-0.491,0.538)$ and adjusted analysis $a b=-0.075,95 \%$ C.I $(-0.592,0.968)$. This means that if everyone in the study had the 
intervention and patient's participation in decision making increased by the mean difference between the control and intervention group, postoperative pain intensity would not change significantly from baseline.

The indirect effect Path $a * b$, for Figure 3

As expected from the results of Path $a$ and Path $b$ analysis results, the unadjusted path model, gave a significant indirect effect $a b=0.696[0.385$, 1.112]. That means, the indirect effect, of treatment on patient satisfaction, with patient participation in decision making as the potential mediator, was statistically significant. Had everyone had the intervention, the patient satisfaction would have increased significantly from baseline when patient's participation in decision-making increases by it's the mean difference between the control and intervention group. However, the adjusted analysis showed an insignificant indirect effect $0.006,95 \%$ C.I $(-0.709,0.601)$.

Covariance of Path a and Path b estimates: Figure 2

One of the most interesting aspects of multilevel mediation unlike to the usual between subject mediation is the presence of, the covariance of Path $a$ and Path b estimates in the estimation of the indirect effect (see oXjjb in Figure 2 and 3). Both the unadjusted and adjusted estimates were not significantly different from zero, with an estimate of oXjbj $=0.005,95 \%$ C.I $(-0.03,0.076)$ and $囚 j b j=-0.009,95 \%$ C.I $(-0.055,0.082)$ respectively. This indicates that those who had a higher participation in decision making, as a result of the education also do not have a lower worst pain intensity consequently.

Covariance of Path $a$ and Path b estimates: Figure 3

The population variance for this second mediation model was also insignificant. Both the unadjusted oهjbj $=-0.004[-0.047,0.022]$ and adjusted $\sigma$ Xjbj $=0.022,95 \%$ C.I $(-0.051,0.082)$ estimates were statistically insignificant. This means that those who had a higher participation in decision making, because of the education also do not have a higher reported satisfaction. 


\section{Discussion}

A significant difference was observed between the treatment and control group, at least at one measurement point for all outcome measures, except for patients' satisfaction, perceived pain relief and pain interference with sleeping. For these outcomes, no significant difference were observed between the groups. In addition for almost all outcome measures, both the linear mixed effect regression and the doubly robust estimation demonstrated consistent results. The exceptions are only for worst pain intensity, pain interference with sleeping and pain causing the feeling of anxiousness. This is expected as the double robust technique is robust for model misspecification compared to linear regression methods [38]. Also, when covariate imbalances between the treated and control group are large, linear regression is expected to produce a biased estimate, especially when such covariates are also non-linearly associated with the outcome [47].

The other important result observed was that patients' worst pain intensity and pain interference with breathing and coughing were lower at 24 and 48 hours after surgery in the treatment group. Whereas, pain interference with activities on bed and with movement were lower in the treatment group at all measurement points. Outcome measures like patients' least and current level of pain, time spent in severe pain and patient participation in decision making were lower only at 48 hours after surgery. Observing significant effects at later postoperative periods compared to the early time-points could arise from the natural surgical ward contexts in the low resource settings, the nature of preoperative information itself and complex psychological phenomena.

There is a limit to what extent pain management can be successful without the use of strong analgesics. No matter how effective an education is, it is an adjunct treatment [19] and can not replace effective analgesics. At the time of this study, no opioids were available for the surgical patient and Ethiopia is classified as a country with nil morphine per capita [48]. Also, giving patients specific information about the importance of good postoperative analgesia might improve their understanding, however, this does not translate necessarily to better postoperative pain outcome. Psychologists explain this by the difference between automatic and planned behavior [49]. Automatic processes, or habits, enable behaviors to be carried out with a little or no demand for cognitive effort, and they make behavioral changes very complicated [50]. Education, therefore, can lead to improved knowledge; however, this does not necessarily change old beliefs and habits. And it might be possible that patients can have increased knowledge of pain treatment and increases participation, without the desired changes in their beliefs or behaviors in accepting analgesics after surgery [50]. The results of this study should encourage HCPs, or researcher that even without opioids with education and non-pharmacological options of pain management, this study demonstrated that improvement can be achieved at least after 12 hours of the surgery.

The difference between patients' worst level of pain with that of current level of pain and, least level of pain, could be associated with the fact that these intensity measures (least and current) are not as sensitive as worst pain intensity in detecting treatment effects, and authors have been recommending against [51]. A clinical trial in Taiwan also reported no effect of the treatment when the outcome was current level of pain and the average level of pain, instead of worst pain intensity [52]. It is also worthy to mention that a recent RCT from Germany, reported no superiority of preoperative patient education over the standard of care for most of the outcome measures authors used, including postoperative pain intensity [53]. Patients' participation in decision making was notably higher in the treatment group compared to the control at 24 hours after the surgery. This is

Page $14 / 24$ 
expected as we have encouraged patients in the treatment group not to be passive and shy, rather to participate actively in the choice and manner of pain management. The goal of encouraging patients to participate in decision-making is to increase satisfaction and better health outcomes. Studies have also hinted this even can reduce the patient report of pain intensity $[27,54]$ and well randomized controlled trials are also currently investigating the topic [55].

Our results from the mediation analysis, however, revealed insignificant indirect effect, for both pain intensity and patient satisfaction, and patient participation in decision did not mediate the treatment with both outcome measures. Still, our result should not be over-emphasized. The lack of mediating effect could also be due to unmeasured confounder between mediator and outcome and this is very difficult to rule out. Also, the absence of statistically significant mediating effects identified could be due to the study being underpowered to detect these effects, as the mediation analysis was secondary and was not powered for this analysis. However, we have measured the most important predictors of severe postoperative pain as identified from systematic review except for preoperative anxiety level. These also were appropriately tested if the addition of such measured confounder covariates-(age, chronic pain, types of surgery, types of anesthesia and duration of surgery) - affected the mediation and the results were the same. A previous study also showed that higher patientdriven participation in decision-making was associated with lower odds (OR, 0.82; 95\% $\mathrm{Cl}, 0.75-0.89)$ of frequent pain, but was not significantly associated with severity of pain. Interestingly they have found no significant association with either frequency or severity of pain when the patient participation was physiciandriven [56]. Despite, our reported insignificant indirect effect, we encourage patient participation in decision making, as insignificant indirect effect does not mean, no evidence of indirect effect at all. Even statistics aside patient participation in decision making is justified on humane grounds alone [25]. Nevertheless, it is unquestionable that the question how does preoperative education is expected to lower postoperative pain intensity and increase patient satisfaction, should be the focus of future researches. Maybe this will pave the way towards consistent results, when it comes to the impact of preoperative patient education on postoperative pain, and also explain conflicting results on the topic. The focus of the mediation analysis was to test whether our theory of how the intervention worked was correct rather than test a more complex mediation model. Hence, future research could test a more complicated model that includes multiple potential mediators in a single pathway, to show a process of change in several variables as part of the treatment process. Simply testing, whether patient educational intervention is associated with a decreased postoperative pain intensity is not enough and future studies should also establish the causal mechanisms by which educational intervention improves postoperative pain. In this way, others would be benefited in designing their intervention by including the mediating variable responsible for reducing patient pain intensity.

\section{Strength and limitations of the study}

The strength of this study was the large study sample $(n=700)$, with repeated measures, very few missing values and high adherence to treatment. Selection bias as appropriately controlled by powerful statistical methods. In addition, unlike many previous studies, we have attempted to understand how the intervention might have affected the outcome variable using the causal mediation analysis. Also, there are no previous reports which augmented preoperative patient education with HCPs education. All these factors contribute to the design positively and give credence to the findings. However, there some limitations that are worthy to note. There was a clear a baseline imbalance between the control and treatment groups, which could bias results to

Page 15/24 
a larger extent and caution is mandatory when interpreting results. Even though we have dealt this during the treatment effect estimation, it is still of a concern for the internal validity of the study. Another important limitation of this study is that the association between preoperative patient education and pain intensity or patient education and patient satisfaction, the mechanisms underlying this association may be complicated. Future research should consider a number of other potential mediators of the above-mentioned association. Even patient participation in decision making should be tested with a more powerful study designs [57]. Heterogeneous sample from different surgical categories might also affect the results in the same manner. This has been also raised previously as a concern from previous trials dealing the same topic [58]; but it could contribute positively to external validity and generalizability of the study. Aside from this, there are known threats to internal validity when one is implementing a quasi-experiment study design. We have tried to control for most threats using various methods. In this regard, the use of two control groups adequately controlled for what called "history effect" [59]. Maturation also seems not to affect the trial as the duration of the study was short $[11,60]$. Patients are the only one who was blinded so there is a threat of Hawthorne effect [59].

\section{Conclusions}

To sum up, this study has tested the impact of the educational intervention, in decreasing postoperative pain intensity by encouraging patients to be part of the solution (participate in the care). The treatment was successful in increasing patients participation in decision making, as anticipated. However, its impact on decreasing pain intensity was only noted at the last measurement point after surgery. Patient participation in decision making not mediated the treatment with pain intensity, even though demonstrated the same pattern.

\section{Declarations}

\section{Ethics Approval and Consent to Participate}

The study was approved by the Jimma University Institutional Review Board; Ref. No RPGC/06/2016 and Ludwig Maximillian University of Munich Medical Ethics Committee; Ref. No 17-224. All participating hospitals

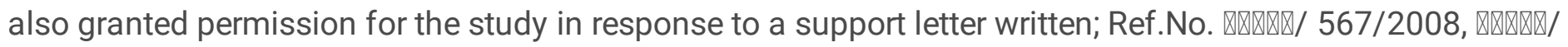

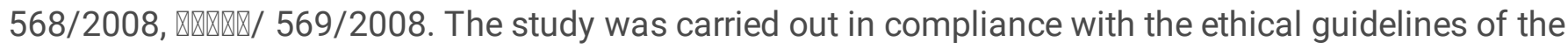
Declaration of Helsinki. A comprehensive oral and written patient information of the purpose and procedure of the study was given. Before inclusion of participants, informed consent was obtained, and patients were informed about their rights to refuse or withdraw at any given time. Confidentiality of the individual information gathered was discussed, and additionally, any personal information was anonymized before the final analysis.

\section{Consent for publication}

Not Applicable

\section{Availability of data and materials}

The datasets used and/or analysed during the current study are available from the corresponding author on reasonable request. 
The authors declare that they have no competing interests

\section{Funding}

This study was supported by the Jimma University and CIHLMU Center for International Health, LudwigMaximilians-Universität, Munich, Germany.

\section{Authors' contributions}

MTE, PIB, MS, MT and DI conceived and designed the study. MTE, AH, YA, GGM contributed to data acquisition and involved supervision of data collection. MTE, PIB, MS, MT and DI analyzed and wrote the paper. PIB, MS, MT and DI critically reviewed the manuscript. All authors read and approved the final manuscript.

\section{Acknowledgments}

We want to thank Jimma University, Department of Anesthesiology, Jimma University Medical Center, Yekatit 12 Hospital Medical College, Zewditu Memorial Hospital, the data collectors, data entry clerks, supervisors, and patients for their cooperation. We are also grateful to Dr. Ursula Berger and Dr. Mulusew Gerbaba for reviewing the statistical analysis. We would like further extend our thanks to the CIHLMU Center for International Health, Ludwig-Maximilians-Universität, Munich, Germany, for its support.

\section{References}

1. Weiser TG, Haynes AB, Molina G, Lipsitz SR, Esquivel MM, Uribe-Leitz T, Fu R, Azad T, Chao TE, Berry WR: Size and distribution of the global volume of surgery in 2012. World Health Organization Bulletin of the World Health Organization 2016, 94(3):201.

2. Weiser TG, Makary MA, Haynes AB, Dziekan G, Berry WR, Gawande AA: Standardised metrics for global surgical surveillance. The Lancet 2009, 374(9695):1113-1117.

3. Crombie IK, Davies HT, Macrae WA: Cut and thrust: antecedent surgery and trauma among patients attending a chronic pain clinic. Pain 1998, 76(1-2):167-171.

4. Gaskin DJ, Richard P: The economic costs of pain in the United States. The Journal of Pain 2012, 13(8):715724.

5. Kuusniemi K, Pöyhiä R: Present-day challenges and future solutions in postoperative pain management: results from PainForum 2014. Journal of Pain Research 2016, 9:25-36.

6. Gerbershagen HJ, Aduckathil S, van Wijck AJ, Peelen LM, Kalkman CJ, Meissner W: Pain intensity on the first day after surgery: a prospective cohort study comparing 179 surgical procedures. Anesthesiology 2013, 118(4):934-944.

7. Gan TJ: Poorly controlled postoperative pain: prevalence, consequences, and prevention. Journal of Pain Research 2017, 10:2287-2298. 
8. Kehlet $\mathrm{H}$, Holte K: Effect of postoperative analgesia on surgical outcome. BJA: British Journal of Anaesthesia 2001, 87(1):62-72.

9. King NB, Fraser V: Untreated Pain, Narcotics Regulation, and Global Health Ideologies. PLOS Medicine 2013, 10(4):e1001411.

10. Murray AA, Retief FW: Acute postoperative pain in 1231 patients at a developing country referral hospital: incidence and risk factors. Southern African Journal of Anaesthesia and Analgesia 2016, 22(1):19-24.

11. Fink C, Diener MK, Bruckner T, Müller G, Paulsen L, Keller M, Büchler MW, Knebel P: Impact of preoperative patient education on prevention of postoperative complications after major visceral surgery: study protocol for a randomized controlled trial (PEDUCAT trial). Trials 2013, 14(1):271.

12. Lilja Y, Rydén S, Fridlund B: Effects of extended preoperative information on perioperative stress: an anaesthetic nurse intervention for patients with breast cancer and total hip replacem. Intensive and Critical Care Nursing 1998, 14(6):276-282.

13. Oshodi TO: The impact of preoperative education on postoperative pain. Part 2. British journal of nursing 2007, 16(13).

14. Devine EC: Effects of psychoeducational care for adult surgical patients: A meta-analysis of 191 studies. Patient education and counseling 1992, 19(2):129-142.

15. Shuldham CM, Fleming S, Goodman $\mathrm{H}$ : The impact of pre-operative education on recovery following coronary artery bypass surgery. A randomized controlled clinical trial. European heart journal 2002, 23(8):666674.

16. Louw A, Diener I, Butler DS, Puentedura EJ: Preoperative education addressing postoperative pain in total joint arthroplasty: review of content and educational delivery methods. Physiotherapy theory and practice 2013, 29(3):175-194.

17. Grinstein-Cohen O, Sarid O, Attar D, Pilpel D, Elhayany A: Improvements and difficulties in postoperative pain management. Orthopaedic Nursing 2009, 28(5):232-239.

18. Zhang Z, Zheng C, Kim C, Van Poucke S, Lin S, Lan P: Causal mediation analysis in the context of clinical research. Annals of translational medicine 2016, 4(21).

19. Chou R, Gordon DB, de Leon-Casasola OA, Rosenberg JM, Bickler S, Brennan T, Carter T, Cassidy CL, Chittenden EH, Degenhardt E: Management of Postoperative Pain: a clinical practice guideline from the American pain society, the American Society of Regional Anesthesia and Pain Medicine, and the American Society of Anesthesiologists' committee on regional anesthesia, executive committee, and administrative council. The Journal of Pain 2016, 17(2):131-157.

20. IASP Interprofessional Pain Curriculum Outline [http://www.iasp-

pain.org/Education/CurriculumDetail.aspx?ItemNumber=2057] 
21. Pain IAftSo: Guide to Pain Management in Low-Resource Settings. In: Educational material written for general distribution to health care providers by a multidisciplinary and multinational team of authors. Edited by Andreas Kopf NBP. SEATTLE; 2010: 317-320.

22. Managing Acute Pain in the Developing World In: Pain Clinical Updates. Edited by Ballantyne JC, vol. XIX: International Association for the Study of Pain; 2011.

23. Health MMo: National Pain Managment Guideline. Ethiopia: . In.: Bole Printing; 2007.

24. Carr-Chellman A, Hoadley C: Introduction to special issue: Instructional systems design and the learning sciences, vol. 44; 2004.

25. Guadagnoli E, Ward P: Patient participation in decision-making. Social Science \& Medicine 1998, 47(3):329339.

26. Karlsten R, Ström K, Gunningberg L: Improving assessment of postoperative pain in surgical wards by education and training. Quality and Safety in Health Care 2005, 14(5):332-335.

27. Meissner W, Coluzzi F, Fletcher D, Huygen F, Morlion B, Neugebauer E, Pérez AM, Pergolizzi J: Improving the management of post-operative acute pain: priorities for change. Current medical research and opinion 2015, 31(11):2131-2143.

28. Practice Guidelines for Acute Pain Management in the Perioperative SettingA Report by the American Society of Anesthesiologists Task Force on Pain Management, Acute Pain Section. Anesthesiology 1995, 82(4):1071-1081.

29. O'Donnell KF: Preoperative Pain Management Education: A Quality Improvement Project. Journal of PeriAnesthesia Nursing 2015, 30(3):221-227.

30. Andersson V, Otterstrom-Rydberg E, Karlsson A-K: The Importance of Written and Verbal Information on Pain Treatment for Patients Undergoing Surgical Interventions. Pain Management Nursing 2015, 16(5):634641.

31. Carr ECJ, Nicky Thomas V, Wilson-Barnet J: Patient experiences of anxiety, depression and acute pain after surgery: a longitudinal perspective. International journal of nursing studies 2005, 42(5):521-530.

32. Gordon DB, Polomano RC, Pellino TA, Turk DC, McCracken LM, Sherwood G, Paice JA, Wallace MS, Strassels SA, Farrar JT: Revised American Pain Society Patient Outcome Questionnaire (APS-POQ-R) for Quality Improvement of Pain Management in Hospitalized Adults: Preliminary Psychometric Evaluation. The Journal of Pain 2010, 11(11):1172-1186.

33. Sousa VD, Rojjanasrirat W: Translation, adaptation and validation of instruments or scales for use in crosscultural health care research: a clear and user-friendly guideline. Journal of evaluation in clinical practice 2011, 17(2):268-274. 
34. Strohbuecker B, Mayer H, Evers GC, Sabatowski R: Pain prevalence in hospitalized patients in a German university teaching hospital. Journal of pain and symptom management 2005, 29(5):498-506.

35. Cheng J, Edwards LJ, Maldonado-Molina MM, Komro KA, Muller KE: Real longitudinal data analysis for real people: building a good enough mixed model. Statistics in medicine 2010, 29(4):504-520.

36. Thabane L, Mbuagbaw L, Zhang S, Samaan Z, Marcucci M, Ye C, Thabane M, Giangregorio L, Dennis B, Kosa $\mathrm{D}$ et al: A tutorial on sensitivity analyses in clinical trials: the what, why, when and how. BMC medical research methodology 2013, 13:92-92.

37. Rothaug J, Zaslansky R, Schwenkglenks M, Komann M, Allvin R, Backström R, Brill S, Buchholz I, Engel C, Fletcher D et al: Patients' Perception of Postoperative Pain Management: Validation of the International Pain Outcomes (IPO) Questionnaire. The Journal of Pain 2013, 14(11):1361-1370.

38. Robins JM, Rotnitzky A, Zhao LP: Estimation of regression coefficients when some regressors are not always observed. Journal of the American statistical Association 1994, 89(427):846-866.

39. Lunceford JK, Davidian M: Stratification and weighting via the propensity score in estimation of causal treatment effects: a comparative study. Statistics in medicine 2004, 23(19):2937-2960.

40. Gupta P, Rettiganti M, Rice TB, Wetzel RC: Impact of 24/7 in-hospital intensivist coverage on outcomes in pediatric intensive care. A multicenter study. American journal of respiratory and critical care medicine 2016, 194(12):1506-1513.

41. Elze MC, Gregson J, Baber U, Williamson E, Sartori S, Mehran R, Nichols M, Stone GW, Pocock SJ: Comparison of Propensity Score Methods and Covariate Adjustment: Evaluation in 4 Cardiovascular Studies. Journal of the American College of Cardiology 2017, 69(3):345-357.

42. Bolger N, Laurenceau J-P: Intensive Longitudinal Methods: An Introduction to Diary and Experience Sampling Research: Guilford Press; 2013.

43. Krull JL, MacKinnon DP: Multilevel modeling of individual and group level mediated effects. Multivariate behavioral research 2001, 36(2):249-277.

44. Mallinckrodt B, Abraham WT, Wei M, Russell DW: Advances in testing the statistical significance of mediation effects. Journal of Counseling Psychology 2006, 53(3):372.

45. Kenny DA, Korchmaros JD, Bolger N: Lower level mediation in multilevel models. Psychological methods 2003, 8(2):115-128.

46. [http://www.page-gould.com/r/indirectmlm/]

47. Golinelli D, Ridgeway G, Rhoades H, Tucker J, Wenzel S: Bias and variance trade-offs when combining propensity score weighting and regression: with an application to HIV status and homeless men. Health Services and Outcomes Research Methodology 2012, 12(2-3):104-118. 
48. Cleary J, Powell RA, Munene G, Mwangi-Powell FN, Luyirika E, Kiyange F, Merriman A, Scholten W, Radbruch $\mathrm{L}$, Torode $\mathrm{J}$ et al: Formulary availability and regulatory barriers to accessibility of opioids for cancer pain in Africa: a report from the Global Opioid Policy Initiative (GOPI). Annals of Oncology 2013, 24(suppl_11):xi14xi23.

49. Ajzen I, Fishbein M: Attitudes and the Attitude-Behavior Relation: Reasoned and Automatic Processes. European Review of Social Psychology 2000, 11(1):1-33.

50. van Dijk JF, Schuurmans MJ, Alblas EE, Kalkman CJ, van Wijck AJ: Postoperative pain: knowledge and beliefs of patients and nurses. J Clin Nurs 2017, 26(21-22):3500-3510.

51. Jensen MP, Castarlenas E, Tomé-Pires $C$, de la Vega $R$, Sánchez-Rodríguez E, Miró J: The number of ratings needed for valid pain assessment in clinical trials: replication and extension. Pain Medicine 2015, 16(9):17641772.

52. Chen SR, Chen CS, Lin PC: The effect of educational intervention on the pain and rehabilitation performance of patients who undergo a total knee replacement. Journal of clinical nursing 2014, 23(1-2):279287.

53. Klaiber U, Stephan-Paulsen LM, Bruckner T, Müller G, Auer S, Farrenkopf I, Fink C, Dörr-Harim C, Diener MK, Büchler MW et al: Impact of preoperative patient education on the prevention of postoperative complications after major visceral surgery: the cluster randomized controlled PEDUCAT trial. Trials 2018, 19(1):288.

54. Brekke M, Hjortdahl P, Kvien TK: Involvement and satisfaction: a Norwegian study of health care among 1,024 patients with rheumatoid arthritis and 1,509 patients with chronic noninflammatory musculoskeletal pain. Arthritis care \& research 2001, 45(1):8-15.

55. McDonall J, Steiger R, Reynolds J, Redley B, Livingston P, Botti M: Patient participation in postoperative care activities in patients undergoing total knee replacement surgery: Multimedia Intervention for Managing patient Experience (MIME). Study protocol for a cluster randomised crossover trial, vol. 17; 2016.

56. Borders TF, Xu KT, Heavner J, Kruse G: Patient involvement in medical decision-making and pain among elders: physician or patient-driven? BMC Health Services Research 2005, 5:4-4.

57. Pirlott AG, MacKinnon DP: Design approaches to experimental mediation. Journal of experimental social psychology 2016, 66:29-38.

58. Shuldham C: 2. Pre-operative education - a review of the research design. International journal of nursing studies 1999, 36(2):179-187.

59. Fife-Schaw C: Quasi-experimental designs. Research methods in psychology 2006:88-103.

60. Cook TD, Campbell DT: Experimental and quasi-experimental designs for generalized causal inference.

\section{Figures}




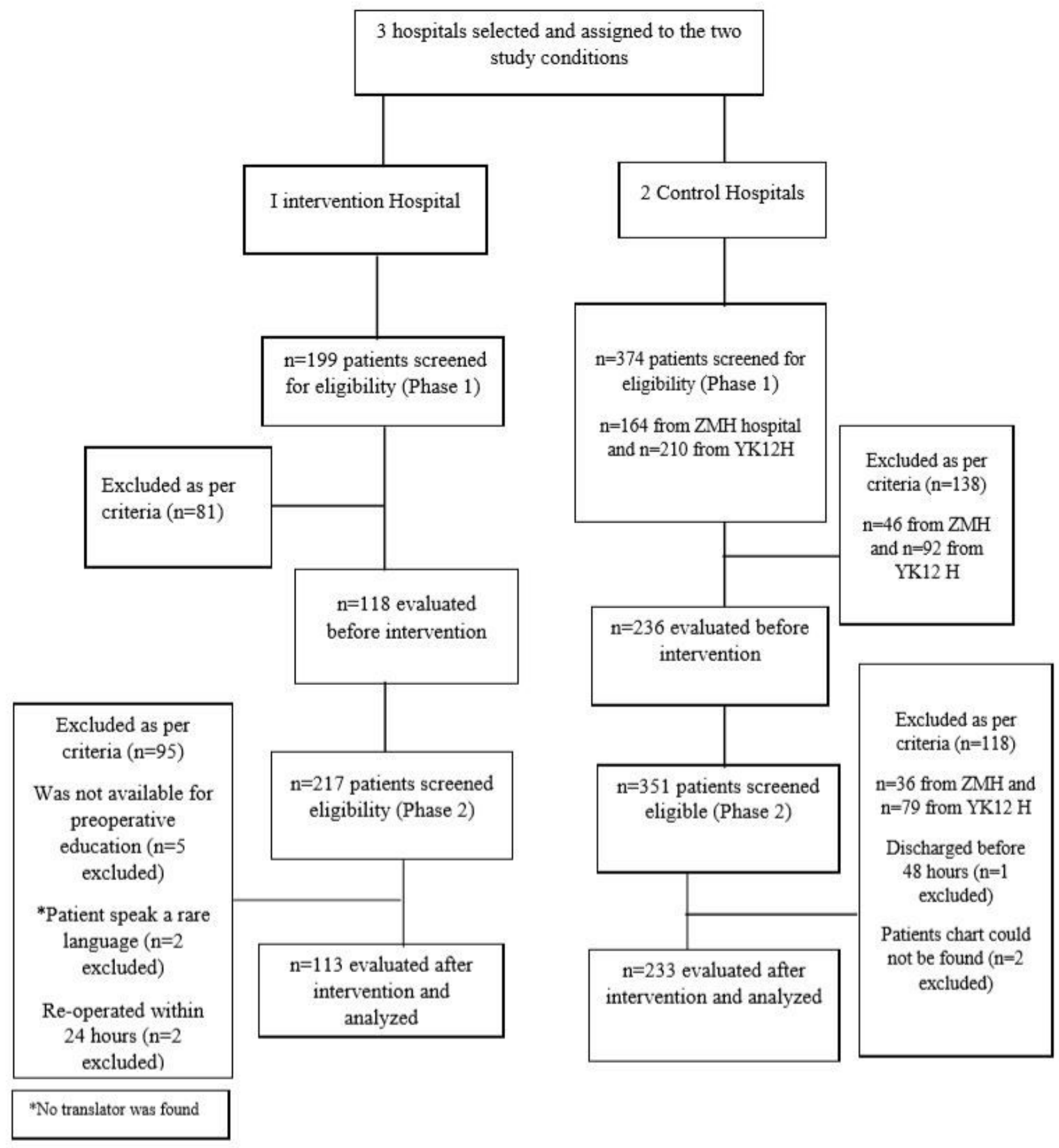

\section{Figure 1}

Participant's flow chart. Phase 1 is the time before the intervention (Bassline), and phase 2 is the time after the baseline. The gap between phase 1 and phase 2 here is about 20 weeks. 


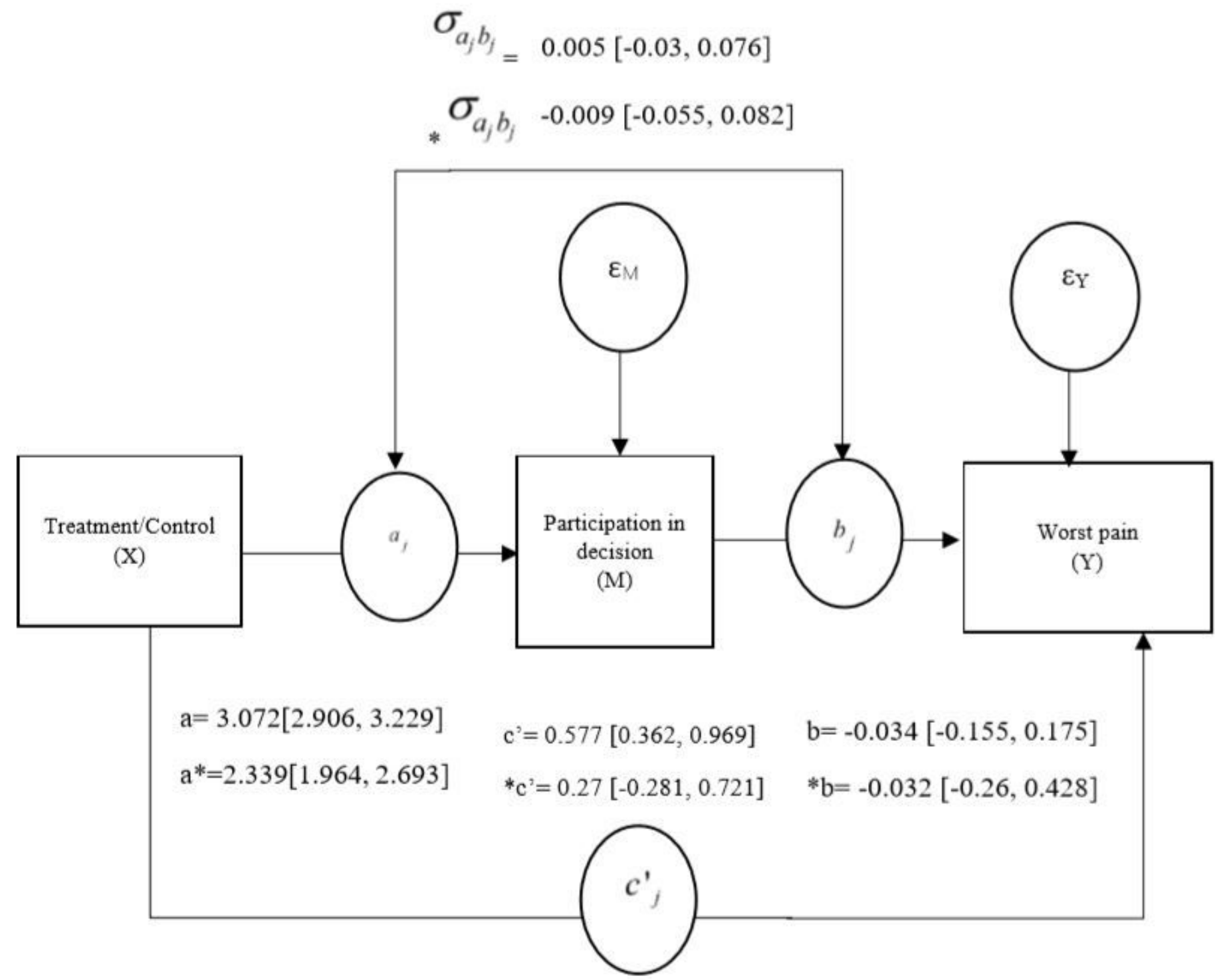

Figure 2

Within-subject mediation (see Bolger and Laurenceau, 2013): Diagram and structural equations. To reduce confusion, we have omitted time as a predictor and we treat $\mathrm{X}, \mathrm{M}$, and $\mathrm{Y}$ as varying within-subjects only. *Adjusted for age, sex, preoperative pain, type of surgery, type of anesthesia, baseline worst pain intensity and duration of surgery. 


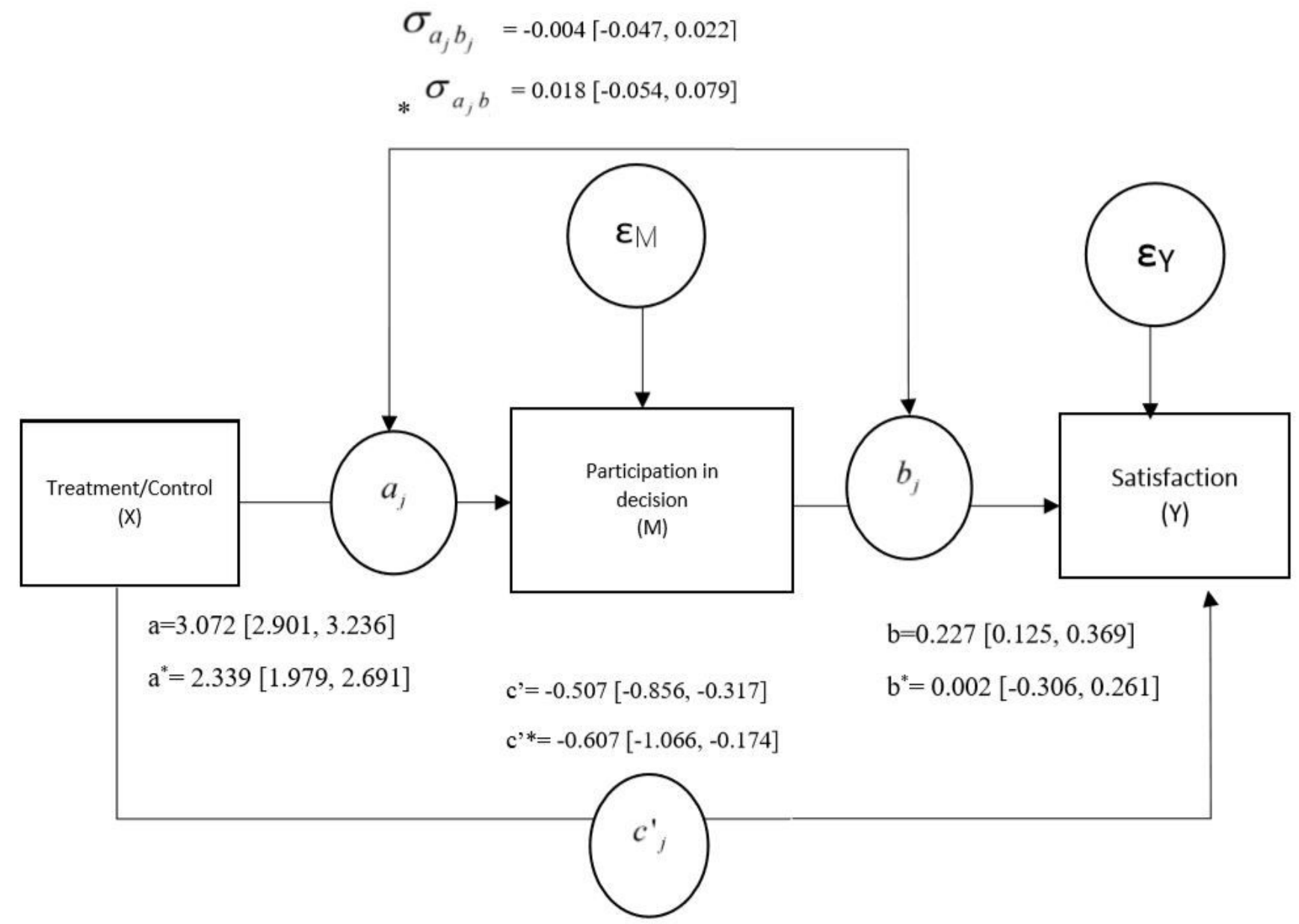

Figure 3

Within-subjects mediation for satisfaction (see Bolger and Laurenceau, 2013): To reduce confusion, we have omitted time as a predictor and we treat $\mathrm{X}, \mathrm{M}$, and $\mathrm{Y}$ as varying within-subjects only. *Adjusted for age, sex, preoperative pain, type of surgery, type of anesthesia, baseline worst pain intensity and duration of surgery. Supplementary Files

This is a list of supplementary files associated with this preprint. Click to download.

- AdditionalFile1.docx 